Check for updates

Cite this: Mater. Adv., 2022, 3, 931

Received 30th September 2021 Accepted 22nd November 2021

DOI: $10.1039 / \mathrm{d} 1 \mathrm{ma} 00909 \mathrm{e}$

rsc.li/materials-advances

\section{Electroless nickel plating on a biomineral-based sponge structure $\uparrow$}

\author{
Hiroaki Takeoka, ${ }^{a}$ Musashi Seike, ${ }^{a}$ Yoshinobu Nakamura, ${ }^{\text {bc }}$ Hiroaki Imai, (D) d \\ Yuya Oaki (D)*d and Syuji Fujii (D) *bc
}

\begin{abstract}
A 3-dimensional polypyrrole-palladium nanocomposite material was prepared by transferring a sponge structure of a sea urchin spine via chemical oxidative polymerization. The nanocomposite material was successfully coated with nickel, maintaining the 3dimensional sponge structure by electroless plating in an aqueous medium.
\end{abstract}

\section{Introduction}

Electroless plating, also known as chemical plating, is one of the industrial processes that allows coating of metal layers, such as nickel (Ni) and copper, onto insulating substrates in a liquid bath. ${ }^{1-3}$ In contrast to electroplating, which requires an externally generated electric current to reduce high-oxidationstate metal ions, electroless plating proceeds based on an autocatalytic chemical reaction. To attain the autocatalytic reaction, an appropriate catalyst (e.g., palladium (Pd) nanoparticles) should be introduced on the surface of the substrates. Electroless Ni plating has been largely applied in the electric materials field to form interconnects in 2-dimensional printed circuit boards. ${ }^{4-6}$ Additionally, electroless plating has been utilized to coat colloidal particles and nanotubes with $\mathrm{Ni}$ toward applications as conducting spacers for electronic devices and additives for electromagnetic absorbing materials. ${ }^{7-13}$ Although electroless plating could be applied to substrates with various shapes, there have been few reports

\footnotetext{
${ }^{a}$ Division of Applied Chemistry, Graduate School of Engineering Osaka Institute of Technology, 5-16-1, Omiya, Asahi-ku, Osaka 535-8585, Japan

${ }^{b}$ Department of Applied Chemistry, Faculty of Engineering Osaka Institute of Technology, 5-16-1 Omiya, Asahi-ku, Osaka 535-8585, Japan.

E-mail: syuji.fujii@oit.ac.jp

'Nanomaterials Microdevices Research Center, Osaka Institute of Technology, 5-16-1 Omiya, Asahi-ku, Osaka 535-8585, Japan

${ }^{d}$ Department of Applied Chemistry, Faculty of Science and Technology, Keio University, 3-14-1 Hiyoshi, Kohoku-ku, Yokohama 223-8522, Japan.

E-mail: oakiyuya@applc.keio.ac.jp

$\dagger$ Electronic supplementary information (ESI) available. See DOI: 10.1039/d1ma00909e
}

regarding electroless Ni plating on 3-dimensional porous structures. The development of electroless $\mathrm{Ni}$ plating on 3dimensional porous structures is crucial to expand the opportunities and diversity of the materials with porous structures as a functional system.

In this communication, electroless Ni plating was conducted on a 3-dimensional polymer-Pd nanocomposite material with a micrometer-scale sponge structure, which was prepared using a biomineral template (Fig. 1). The nanocomposite materials before and after Ni coating have been extensively characterized in terms of their morphology and chemical compositions using a wide range of analytical techniques such as optical microscopy, Fourier transform infrared spectrometry (FT-IR), scanning electron microscopy (SEM), energy dispersive X-ray spectroscopy (EDX), CHN analysis, and thermogravimetric analysis (TGA).

\section{Results and discussion}

The goal of this article is to coat a material with a sponge structure by Ni via electroless plating. It is therefore important to prepare catalyst-loaded materials with sponge structures. To this end, we first prepared 3-dimensional polymer-Pd nanocomposite materials with the sponge structures by chemical oxidative polymerization of pyrrole (Py) with Pd(II) chloride $\left(\mathrm{PdCl}_{2}\right)$ using a sea urchin spine as a porous biomineral template. Recently, we reported successful syntheses of polypyrrole (PPy)-Pd (PPy-Pd) nanocomposite materials in a onestep manner by aqueous chemical oxidative polymerization of Py using $\mathrm{PdCl}_{2} \cdot{ }^{14,15}$ Here, Py worked as a reductant and $\mathrm{PdCl}_{2}$ functioned as both an oxidant and a source of metal atoms, yielding PPy-Pd metal nanocomposites. Furthermore, the Pd nanoparticles embedded in the PPy matrix were demonstrated to work as effective catalysts for aerobic oxidative homocoupling reactions, ${ }^{16}$ Suzuki-Miyaura cross coupling reactions, ${ }^{17}$ and electroless plating. ${ }^{10}$ The sea urchin spines, composed of $\mathrm{CaCO}_{3}$ nanocrystals, are well known to have a microporous 

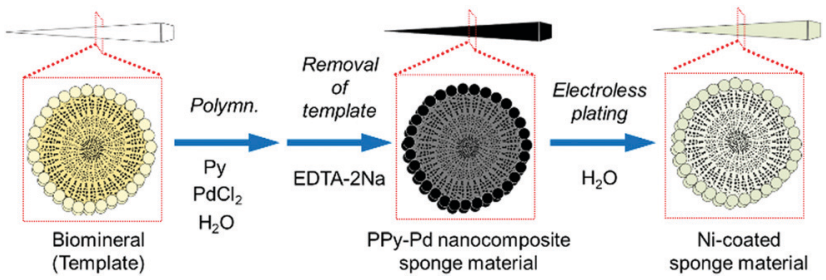

Fig. 1 Scheme illustrating the synthesis of the polypyrrole-Pd (PPy-Pd) nanocomposite sponge material via chemical oxidative polymerization using a biomineral as a template and electroless plating to fabricate the nickel (Ni)-coated sponge material.

structure and have been utilized as a sacrificial template to form functional materials. ${ }^{18-22}$ SEM images showed that the spine had a sponge structure with a pore size of $5 \mu \mathrm{m}-23 \mu \mathrm{m}$ (Fig. 2a-d).

After immersion of one sea urchin spine $(6.3 \pm 2.8 \mathrm{mg})$ in Py monomer for 24 hours, the spine was wiped using a paper towel to remove excess Py monomer before immersion in an aqueous $\mathrm{PdCl}_{2}$ solution. The weight measurements of the spine before and after the immersion in Py monomer indicated the specific pore volume of $0.146 \mathrm{~cm}^{3} \mathrm{~g}^{-1}$, which was of similar value to those reported for the 3-hexylthiophene and 3,4ethylenedioxythiophene system $\left(0.1-0.15 \mathrm{~cm}^{3} \mathrm{~g}^{-1}\right){ }^{21,23}$ Considering the specific pore volume estimated using degassed water after five evacuation and pressurization cycles to remove all gases that might hide in the pores was $0.127 \mathrm{~cm}^{3} \mathrm{~g}^{-1}$, the Py was expected to fill the internal pore space within the spine. Once the Py-containing spine was placed in the aqueous solution of $\mathrm{PdCl}_{2}$ and $\mathrm{NaCl}\left(\mathrm{PdCl}_{2}, 80 \mathrm{mg}\right.$; $\mathrm{NaCl}, 0.160 \mathrm{~g}$; water, $10.0 \mathrm{~g}$ ) at $50{ }^{\circ} \mathrm{C}$, the polymerization started smoothly and the color of the spine changed from white to black, indicating the generation of the $\mathrm{PPy}-\mathrm{Pd}$ nanocomposite. The polymerization was expected to occur first at the surface of the sea urchin spine where the Py monomer and oxidant could contact and then progressed internally via diffusion of the oxidant into the pore. During the polymerization for 1 week with magnetic stirring at

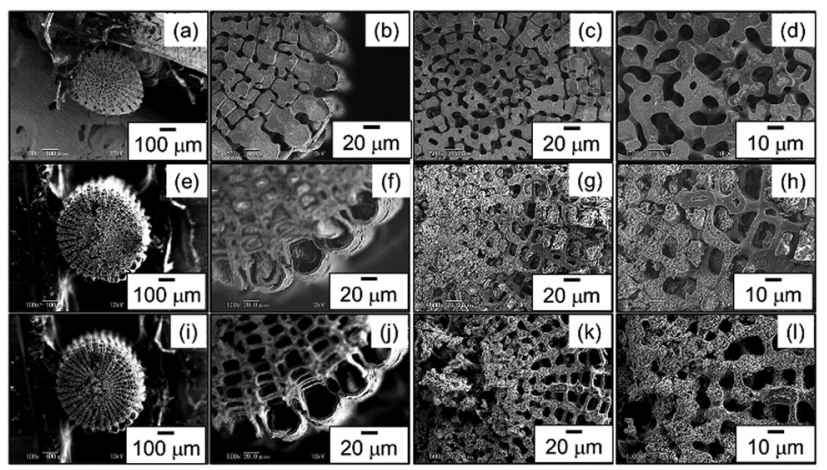

Fig. 2 SEM images of the $(a-d)$ biomineral, $(e-h)$ biomineral/PPy-Pd nanocomposite and ( $\mathrm{i}-\mathrm{l}) \mathrm{PPy}-\mathrm{Pd}$ nanocomposite sponge material obtained by extraction of biomineral from the biomineral/PPy-Pd nanocomposite using EDTA-2Na. ( $b$ and $c$ ), ( $f$ and $g$ ) and ( $j$ and $k$ ) are magnified images of outer and center areas of (a), (e) and (i), respectively. (d), (h) and $(\mathrm{l})$ are magnified images of $(\mathrm{c}),(\mathrm{g})$ and $(\mathrm{k})$, respectively. (a) (i) (ii) (iii) (iv) (v) (vi)

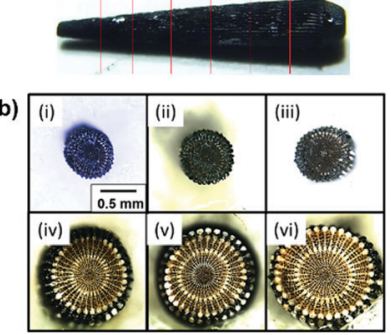

(c)



Fig. 3 (a) Optical photograph and (b) cross-sectional optical micrographs of the biomineral after chemical oxidative polymerization of pyrrole with $\mathrm{PdCl}_{2}$ oxidant. (c) Relationship between cross-sectional area and PPy-Pd nanocomposite coated area.

$200 \mathrm{rpm}$, the PPy-Pd nanocomposite should precipitate within the pores to fill the inner space and onto the surface of the sea urchin spine. Subsequent inspection of the cross section of the resulting sea urchin spine using optical photography revealed that black color was observed relatively homogeneously from the circumference to the center of the cross-sectional circle for the spine with cross-sectional areas of $<5.1 \times 10^{-3} \mathrm{~cm}^{2}$ and the percentage of black-color occupying areas was determined to be $>82 \%$ (Fig. 3). On the other hand, the black color could be observed only on the circumference layer of the crosssectional circle for the spine with cross-sectional areas of $>11.2 \times 10^{-3} \mathrm{~cm}^{2}$ and the percentage of black-color occupying areas was determined to be $<22 \%$ (Fig. 3). The reason is unclear, but there were two possible reasons for this incomplete deposition of the PPy-Pd nanocomposite: (1) $\mathrm{Pd}^{2+}$ oxidant could not diffuse to the center part of the spine through the generated PPy-Pd nanocomposite and (2) Py monomer did not exist in the center part because of elution of the Py monomer into the aqueous phase during the polymerization. Hereafter, the spine/PPy-Pd nanocomposite materials with cross-sectional areas of $<5.1 \times 10^{-3} \mathrm{~cm}^{2}$ were characterized. The SEM images suggest that the pore of the spine was filled with the PPy-Pd nanocomposite (Fig. 2e-h). Fig. 4a-c depict FT-IR spectra of the bare spine template, the PPy-Pd nanocomposite (synthesized by aqueous precipitation polymerization in the absence of spine), and the sea urchin spine/PPy-Pd nanocomposite material. The spectrum for the bare spine was typical of that for the $\mathrm{CaCO}_{3}$. The broad absorption with a peak top of $3401 \mathrm{~cm}^{-1}$ could be assigned to the stretching vibration and asymmetric stretching vibration of the $\mathrm{O}-\mathrm{H}$ bond, and could be also due to the hydroxy groups and absorbed water on the $\mathrm{CaCO}_{3}$ surface. There were other characteristic peaks at 714 and 876 and $1437 \mathrm{~cm}^{-1}$ corresponding to in-plane bending $\left(\nu_{4}\right)$ and out-ofplane bending $\left(\nu_{2}\right)$ vibrations and asymmetric stretching vibration $\left(\nu_{3}\right)$ of carbonate groups, respectively. ${ }^{24,25}$ The combination bands were also detected at 1796, 2515 and $2872 \mathrm{~cm}^{-1}$ for $\nu_{1}+\nu_{4}, 2 \nu_{2}+\nu_{4}$ and $2 \nu_{3}$, respectively. ${ }^{24}$ In the spectrum of the PPy-Pd nanocomposite, characteristic bipolaron bands at 1222 and $937 \mathrm{~cm}^{-1}$ and broad bands at $1568 \mathrm{~cm}^{-1}(\mathrm{C}-\mathrm{C}$ stretching vibration in Py ring) and $1051 \mathrm{~cm}^{-1}(\mathrm{C}-\mathrm{H}$ and $\mathrm{N}-\mathrm{H}$ in-plane deformation vibration) were observed, indicating the 


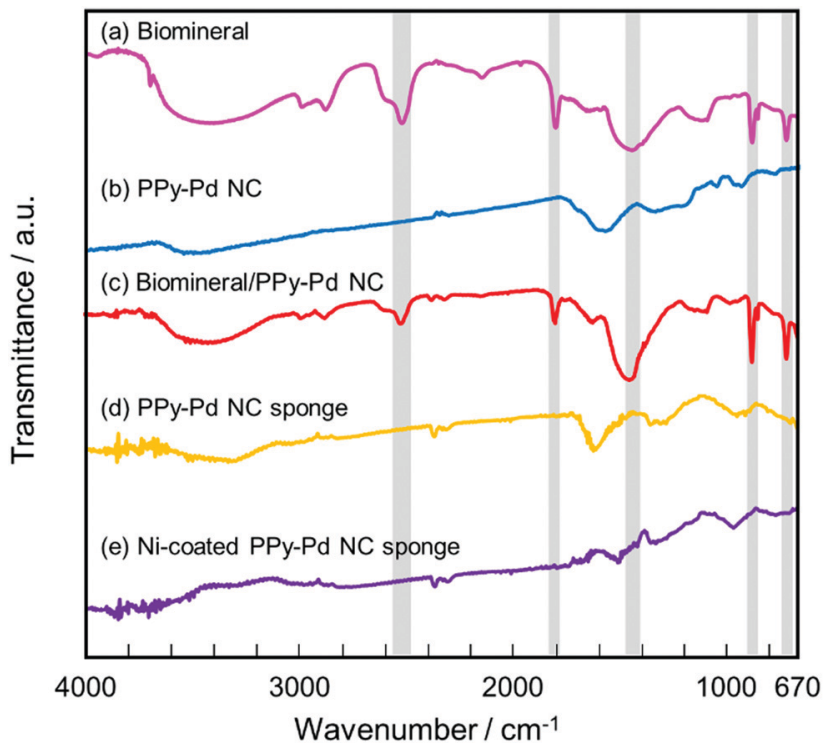

Fig. 4 FT-IR spectra obtained for the (a) biomineral, (b) PPy-Pd nanocomposite bulk powder, (c) biomineral/PPy-Pd nanocomposite, (d) PPy$\mathrm{Pd}$ nanocomposite sponge material and (e) Ni-coated PPy-Pd nanocomposite sponge material.

generation of doped PPy. ${ }^{26}$ In the spectrum of the spine/PPy-Pd nanocomposite, characteristic absorptions attributed to both the $\mathrm{CaCO}_{3}$ and PPy components were observed. CHN analyses gave us meaningful information on chemical composition. Percentages of carbon, hydrogen and nitrogen were determined to be $11.8 \%, 0.31 \%$ and $\sim 0 \%$ for the spine, respectively. PPyPd nanocomposite loading to the spine was determined to be $1.67 \mathrm{wt} \%$, by comparing the nitrogen content of $0.12 \%$ for the spine/PPy-Pd composite material to that of $7.18 \%$ for the PPyPd nanocomposite. (Here, the PPy-Pd nanocomposite loading could be estimated based on nitrogen content, because the spine does not contain nitrogen.) An EDX study, which can map elements, offered worthwhile information on the morphology of the spine/PPy-Pd nanocomposite material (Fig. 5a-c, Fig. S1 and S2, ESI $\dagger$ ). The EDX images confirmed the existence of Ca and Pd elements, which originated from the biomineral and the PPy-Pd nanocomposite, respectively. Interestingly, the locations of the biomineral and PPy-Pd nanocomposite did not overlap, and they existed separately, indicating that the PPy-Pd nanocomposite precipitated in the biomineral pore and filled the pore.

The PPy-Pd nanocomposite could keep its porous structure even after removal of the biomineral using ethylenediaminetetraacetic acid disodium salt (EDTA-2Na), indicating the successful role of the sea urchin spine as a sacrificial template. The SEM image suggested that the thickness of the PPy-Pd wall was $4 \mu \mathrm{m}-28 \mu \mathrm{m}$, which accorded well with the pore size of the original spine (Fig. 2i-l). Transmission electron microscopy (TEM) studies on the ground sample confirmed that the Pd nanoparticles with a diameter of $2-5 \mathrm{~nm}(3 \pm 1 \mathrm{~nm})$ were embedded in the PPy matrix (Fig. S3, ESI $\dagger$ ). The weight loss profile obtained by TGA for the PPy-Pd nanocomposites
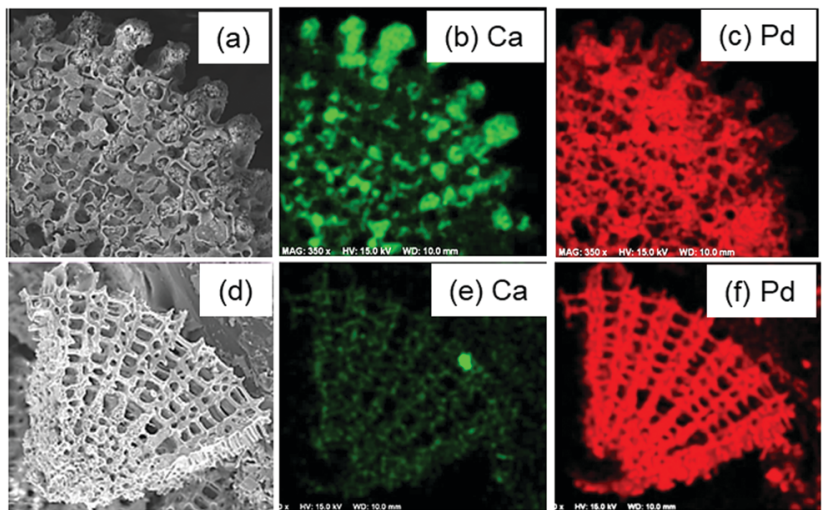

Fig. 5 (a and d) SEM images and ( $b, c, e$, and f) EDX images of $(a-c)$ the biomineral/PPy-Pd nanocomposite and $(d-f)$ the PPy-Pd nanocomposite sponge material obtained by extraction of the biomineral from the biomineral/PPy-Pd nanocomposite using EDTA-2Na.

synthesized in the presence of the spine was in good accordance with that for the PPy-Pd nanocomposites synthesized in the absence of the spine (Fig. S4, ESI $\dagger$ ). This result should indicate that the chemical compositions of these PPy-Pd nanocomposites were the same, which was also supported by FT-IR studies (Fig. 4b and d). The PPy-Pd nanocomposite consisted of $38.6 \mathrm{wt} \%$ PPy and $61.4 \mathrm{wt} \%$ Pd components, which was estimated by comparing the nitrogen content to that of chlorine-doped PPy homopolymer synthesized by aqueous chemical oxidative precipitation polymerization using $\mathrm{FeCl}_{3}$ oxidant, assuming that the PPy component in the PPy-Pd nanocomposite and the PPy homopolymer have the same chemical structure. (CHN analysis indicated $\mathrm{C} / \mathrm{H} / \mathrm{N}$ weight ratios for $\mathrm{PPy}-\mathrm{Cl}$ and $\mathrm{PPy}-\mathrm{Pd}$ nanocomposite were 48/3.19/ 13.97 and 48/3.26/13.80, which accorded well with those theoretically calculated $(48 / 3 / 14)$, respectively. The hydrogen percentages experimentally determined were a little bit higher than the theoretical values. This might be due to introduction of pyrrolidine units into PPy during the polymerization. ${ }^{27}$ ) This weight ratio was in good agreement with the theoretical value estimated based on the reaction stoichiometry (PPy, $38.3 \mathrm{wt} \%$; $\mathrm{Pd}, 61.7 \mathrm{wt} \%){ }^{14}$ These results suggest that the Py was polymerized with the $\mathrm{Pd}^{2+}$ oxidant in a quantitative manner. The EDX studies showed the existence of Pd element and appreciable attenuation of $\mathrm{Ca}$ element, indicating removal of the biomineral template (Fig. 5d-f and Fig. S2, ESI $\dagger$ ).

$\mathrm{Ni}$ was then deposited on the PPy-Pd nanocomposite sponge material by electroless plating using Pd nanoparticles as a catalyst in an aqueous medium. Ni plating was conducted at $45{ }^{\circ} \mathrm{C}$ for 7 days under magnetic stirring at $250 \mathrm{rpm}$ by placing the PPy-Pd nanocomposite material $(2.2 \mathrm{mg})$ in the Ni-plating solution. The Ni-plating solution consists of Ni(II) sulfate hexahydrate $(3.0 \mathrm{~g})$, sodium phosphinate monohydrate $(1.0 \mathrm{~g})$, sodium acetate $(1.0 \mathrm{~g})$ and deionized water $(98 \mathrm{~mL})$. Prior to the electroless plating, the PPy-Pd nanocomposite material was pre-wetted with methanol. This procedure was required to infiltrate the plating solution into the pore of the nanocomposite material by improvement of the wettability. (Note that the 



Fig. 6 (a and b) Optical micrographs, (c-e) SEM and ( $f-h$ ) EDX images of the Ni-coated PPy-Pd nanocomposite sponge material. (d) and (e) are magnified images of outer and center areas of $(c)$. ( $f-h)$ are EDX images of (c).

contact angles of water and methanol measured just after placing the droplet on the pressed pellet made from PPy were $50^{\circ}$ and $17^{\circ}$, respectively, suggesting that methanol could wet the PPy-Pd nanocomposite better than water.) The resulting Nicoated PPy-Pd nanocomposite sponge material was purified via 5 times replacements of aqueous media with deionized water. After the electroless plating, the color of the material turned from black to metallic, indicating surface coating with $\mathrm{Ni}$ (Fig. 6a and b). The FT-IR spectrum of the Ni-coated nanocomposite material showed fewer characteristic features, because of coating with the metal component (Fig. 4e). SEM images revealed that the Ni was deposited on the surface of the PPyPd nanocomposite sponge material (Fig. 6c-e). Interestingly, the Ni coating thickness was inhomogeneous: the PPy-Pd nanocomposite wall was covered by a Ni overlayer $(>1 \mu \mathrm{m}$ thickness) in some parts (Fig. 6d) and the pores of the PPy-Pd nanocomposite sponge material were filled with $\mathrm{Ni}$ in other parts (Fig. 6e). The reason for this inhomogeneous Ni coating is unclear, but the concentration gradient of $\mathrm{Ni}^{2+}$ ions in the sponge structure generated during the electroless deposition could play some role. Ostwald ripening, which causes size distribution change of deposited $\mathrm{Ni}$ particles through the dissolution and re-deposition during the deposition time, could be another reason. ${ }^{28}$ The Ni-coated PPy-Pd nanocomposite sponge material could keep the 3D structure over five years.

EDX images for the Pd and Ni elements are depicted in Fig. $6 \mathrm{f}-\mathrm{h}$. The EDX images revealed that Pd and Ni elements existed homogeneously, suggesting the successful Ni deposition on the PPy-Pd material and remaining of the $\mathrm{Pd}$ catalyst after the electroless plating. There were areas where the position of Pd was the same as that of Ni. Considering that the size of primary PPy nuclei generated by chemical oxidative polymerization was reported to be approximately $5-10 \mathrm{~nm},{ }^{29}$ the nanocomposite should have a structure in which Pd nanoparticles were embedded in the PPy nanoscale porous matrix. Therefore, small ionic species $\left(\mathrm{Ni}^{2+}\right)$ were expected to diffuse and to contact the Pd nanoparticles, resulting in electroless $\mathrm{Ni}$ plating occurrence on and within the nanocomposite substrate. The atomic ratio of Ni/Pd was determined to be $80.35 / 19.65$ (Fig. S2, ESI $\dagger$ ). The signal assigned to $\mathrm{P}$ element was also detected after Ni plating, which could be due to the generation of a Ni-P alloy via reduction of phosphorous acid. ${ }^{30,31}$

\section{Conclusions}

In conclusion, the PPy-Pd nanocomposite material was prepared by transferring the sponge structure of the biomineral via chemical oxidative polymerization. The 3-dimensional porous PPy-Pd nanocomposite material was then successfully covered with $\mathrm{Ni}$ via aqueous electroless plating utilizing a Pd nanoparticle catalyst embedded in the PPy matrix. Noteworthily, the fabrication of the metal-coated porous material can be conducted entirely in aqueous media. As a biomineral porous template, nacre, eggshell and coral could also be utilized. ${ }^{22}$ Other polymer-Pd nanocomposites, like poly(3,4-ethylenedioxythiophene)-Pd, could be applicable as the template, which have been already synthesized by one-step aqueous chemical oxidative polymerization successfully. ${ }^{32}$ Potential applications for the metal-coated sponge materials include catalyst supports and novel electronic and optical devices. ${ }^{33-35}$ The method developed in this study should widen routes to coat materials with various 3-dimensional structures with metal.

\section{Materials and methods}

\section{Materials}

Unless otherwise stated, all materials were guaranteed reagent grade. Palladium(II) chloride $\left(\mathrm{PdCl}_{2}, 99.9 \%\right)$ and sodium phosphinate monohydrate $\left(\mathrm{NaH}_{2} \mathrm{PO}_{2} \cdot \mathrm{H}_{2} \mathrm{O}, 82.0-86.5 \%\right)$ were obtained from Wako Chemicals. Sodium chloride $(\mathrm{NaCl}$, 99.5\%), aluminium oxide (activated, basic, Brockmann 1, standard grade, $\sim 150$ mesh, $58 \AA$ ), nickel(II) sulfate hexahydrate ( $\geq 99.99 \%$ metal basis), methanol (99.5\%) and sodium acetate (99\%) were obtained from Sigma-Aldrich and were used without further purification. Pyrrole (Py, 98\%) was also obtained from Sigma-Aldrich and purified by passing through a column of activated basic alumina prior to storage at $-15{ }^{\circ} \mathrm{C}$ before use. Sodium hypochlorate $(\mathrm{NaClO})$ aqueous solution $(5 \mathrm{wt} \%)$ was obtained from Kanto Chemical Co. Inc. Deionized water $\left(<0.06 \mu \mathrm{S} \mathrm{cm}^{-1}\right)$ was prepared using deionized water producing apparatus (Advantec MFS RFD240NA: GA25A-0715) and was used for syntheses and purification of materials. A sea urchin spine (Echinometra mathaei) was immersed in $\mathrm{NaClO}$ aqueous solution for $48 \mathrm{~h}$ after ultrasound irradiation for $1 \mathrm{~h}$. Then, the sample was rinsed by a large volume of purified water and then treated at $450{ }^{\circ} \mathrm{C}$ for $4 \mathrm{~h}$ under an air atmosphere. These treatments were performed to remove the incorporated biological macromolecules, according to the previous reports. ${ }^{23}$ 


\section{Observation with a stereoscopic microscope}

The sea urchin spine, PPy-Pd nanocomposite, sea urchin spine/ PPy-Pd nanocomposite, $\mathrm{PPy}-\mathrm{Pd}$ nanocomposite sponge material and the Ni-coated PPy-Pd nanocomposite sponge material were observed using a stereomicroscope (Shodensha Co., Ltd., TG300PC3 fitted with GR 300 BCM 2 camera).

\section{TEM study}

The dilute dispersions of the PPy-Pd nanocomposite dried on a collodion-coated copper grid was observed using TEM (FEI Tecnai G2) operating at $200 \mathrm{kV}$.

\section{SEM study}

The SEM (Keyence VE-8800, $12 \mathrm{kV}$ ) studies were conducted on Au sputter-coated (Elionix SC-701 Quick Coater) dried samples.

\section{Chemical composition}

The PPy-Pd nanocomposite, PPy and Pd loadings of the synthesized materials were determined by comparing the nitrogen contents obtained by CHN analyses (Yanaco CHN-Corder MT-5) with those of the PPy-Pd nanocomposite and the PPy bulk powders prepared by precipitation polymerization. The elemental analyser determines $\mathrm{CHN}$ by total combustion in a stream of $\mathrm{O}_{2} / \mathrm{He}$ followed by adsorbents for by-products due to halogen and sulfur content and determination of the thermal conductivity of the gas stream before and after it passes an adsorbent for water and an adsorbent for carbon dioxide.

\section{FT-IR spectroscopy}

The sea urchin spine, PPy-Pd nanocomposite, sea urchin spine/ PPy-Pd nanocomposite, PPy-Pd nanocomposite sponge material and Ni-coated $\mathrm{PPy}-\mathrm{Pd}$ nanocomposite sponge material dispersed in $\mathrm{KBr}$ discs were studied by FT-IR measurements using an IR Prestige-21 (Shimadzu Co. Ltd) and the LabSolutions IR software. The measurements were conducted under the conditions of 512 scans per spectrum at $4 \mathrm{~cm}^{-1}$ resolution.

\section{EDX spectroscopy study}

Spatial elemental analysis of the materials was performed by field emission SEM (FE-SEM, FEI Sirion) equipped with an EDX microanalyzer (Bruker x-flash) operating at $15 \mathrm{kV}$.

\section{TGA study}

In the TGA (SII, TG/DTA 6300) analysis, dried samples were heated up to $800{ }^{\circ} \mathrm{C}$ under nitrogen at a heating rate of $10{ }^{\circ} \mathrm{C} \min ^{-1}$.

\section{Author contributions}

Hiroaki Takeoka: methodology, investigation. Musashi Seike: methodology, investigation. Yoshinobu Nakamura: methodology, investigation. Hiroaki Imai: methodology, investigation. Yuya Oaki: conceptualization, methodology, investigation, writing - review and editing, supervision, and project administration. Syuji Fujii: conceptualization, methodology, writing - original draft, writing - review and editing, supervision, project administration, and funding acquisition.

\section{Conflicts of interest}

There are no conflicts to declare.

\section{Acknowledgements}

This work was supported by a Grant-in-Aid for Scientific Research (B) (JSPS KAKENHI Grant Number JP 20H02803) and Scientific Research on Innovative Areas "Engineering Neo-Biomimetics (JSPS KAKENHI Grant Number JP15H01602)" and "New Polymeric Materials Based on Element-Blocks (JSPS KAKENHI Grant Number JP15H00767)".

\section{Notes and references}

1 R. C. Agarwala and V. Agarwala, Sadhana, 2003, 28, 475-493. 2 J. Sudagar, J. Lian and W. Sha, J. Alloys Compd., 2013, 571, 183-204.

3 C. A. Loto, Silicon, 2016, 8, 177-186.

4 I. Ohno, Mater. Sci. Eng., A, 1991, 146, 33-49.

5 X. Zhang and J. Zhang, RSC Adv., 2016, 6, 30695-30698.

6 H. Yabu, Y. Hirai and M. Shimomura, Langmuir, 2006, 22, 9760-9764.

7 P. Tierno and W. A. Goedel, J. Phys. Chem. B, 2006, 110, 3043-3050.

8 M. Sanles-Sobrido, M. Bañobre-López, V. Salgueiriño, M. A. Correa-Duarte, B. Rodríguez-González, J. Rivas and L. M. Liz-Marzán, J. Mater. Chem., 2010, 20, 7360-7365.

9 W. Li, T. Qiu, L. Wang, S. Ren, J. Zhang, L. He and X. Li, ACS Appl. Mater. Interfaces, 2013, 5, 883-891.

10 S. Fujii, H. Hamasaki, H. Takeoka, T. Tsuruoka, K. Akamatsu and Y. Nakamura, J. Colloid Interface Sci., 2014, 430, 47-55.

11 Y. Ma, L. Wang, Q. Ye, H. Qin and Q. Fu, Chem. Lett., 2021, 50, 184-186.

12 J. Huang and Z. Chen, RSC Adv., 2017, 7, 25622-25626.

13 R. Zhou, F.-K. Tang, R. W.-Y. Sun, M. K. Tse, Y. Chen, A. S. C. Chan, S. Chen, X. Zhu and K. C.-F. Leung, Mater. Adv., 2021, 2, 236-240.

14 S. Fujii, S. Matsuzawa, Y. Nakamura, A. Ohtaka, T. Teratani, K. Akamatsu, T. Tsuruoka and H. Nawafune, Langmuir, 2010, 26, 6230-6239.

15 S. Fujii, S. Matsuzawa, H. Hamasaki, Y. Nakamura, A. Bouleghlimat and N. J. Buurma, Langmuir, 2012, 28, 2436-2447.

16 A. Bouleghlimat, M. Othman, L. Lagrave, S. Matsuzawa, Y. Nakamura, S. Fujii and N. Buurma, Catalysts, 2017, 7, 280.

17 A. Ohtaka, Y. Kono, T. Teratani, S. Fujii, S. Matsuzawa, Y. Nakamura and R. Nomura, Catal. Lett., 2011, 141, 1097-1103.

18 R. J. Park and F. C. Meldrum, Adv. Mater., 2002, 14, 1167-1169. 
19 C. S. Gaddis and K. H. Sandhage, J. Mater. Res., 2011, 19, 2541-2545.

20 Y. Oaki, M. Kijima and H. Imai, J. Am. Chem. Soc., 2011, 133, 8594-8599.

21 Y. Munekawa, Y. Oaki and H. Imai, Langmuir, 2014, 30, 3236-3242.

22 A. Göppert and H. Cölfen, RSC Adv., 2018, 8, 33748-33752.

23 M. Kijima, Y. Oaki, Y. Munekawa and H. Imai, Chem. - Eur. J., 2013, 19, 2284-2293.

24 T. Z. Forbes, A. V. Radha and A. Navrotsky, Geochim. Cosmochim. Acta, 2011, 75, 7893-7905.

25 A. Barhoum, H. Rahier, R. E. Abou-Zaied, M. Rehan, T. Dufour, G. Hill and A. Dufresne, ACS Appl. Mater. Interfaces, 2014, 6, 2734-2744.

26 R. B. Bjorklund and B. Liedberg, J. Chem. Soc., Chem. Commun., 1986, 1293-1295.

27 S. Rapi, V. Bocchi and G. P. Gardini, Synth. Met., 1988, 24, 217-221.
28 A. Kregar, A. Kravos and T. Katrašnik, Fuel Cells, 2020, 20, 487-498.

29 S. P. Armes, M. Aldissi, M. Hawley, J. G. Beery and S. Gottesfeld, Langmuir, 1991, 7, 1447-1452.

30 A. Lelevic and F. Walsh, Surf. Coat. Technol., 2019, 369, 198-220.

31 A. Brenner, Electrodeposition of alloys: principles and practice, Academic Press, New York, 1963.

32 H. Hamasaki, Y. Maekawa, S. Matsuzawa, A. Ohtaka, Y. Nakamura and S. Fujii, Chem. Lett., 2012, 41, 1658-1659.

33 Y. Gong, S. Zhang, H. Gao, Z. Ma, S. Hu and Z. a. Tan, Sustainable Energy Fuels, 2020, 4, 4415-4458.

34 F. Han, X. Su, M. Huang, J. Li, Y. Zhang, S. Zhao, F. Liu, B. Zhang, Y. Wang, G. Zhang, R. Sun and C.-P. Wong, J. Mater. Chem. C, 2018, 6, 8135-8143.

35 F. Carlesso, L. E. A. Vieira, L. A. Berni, G. d. S. Savonov, A. Remesal Oliva, W. Finsterle and E. L. de Miranda, Astrophys. J., Suppl. Ser., 2020, 248, 4. 